\title{
Helmholtz-Manakov Solitons
}

\author{
J. M. Christian and G. S. McDonald, \\ Joule Physics Laboratory, School of Computing, Science and Engineering, Institute of Materials Research, \\ University of Salford, Salford M5 4WT, U.K. \\ P. Chamorro-Posada, \\ Departmento de Teoría de la Señal y Comunicaciones e Ingeniería Telemática, Universidad de Valladolid, \\ ETSI Telecomunicación, Campus Miguel Delibes s/n, 47011 Valladolid, Spain.
}

\section{Introduction}

The propagation of a spatial vector soliton beam in a Kerr planar waveguide is typically described by the Manakov equation [1]. However, the assumption of beam paraxiality breaks the rotational symmetry of the wave propagation problem. Manakov-based descriptions are, for example, incapable of describing physical effects associated with off-axis propagation at non-trivial angles. We will report the first Helmholtz generalizations of the Manakov equation and its soliton solutions, along with a thorough investigation of the dynamical properties of the new solutions.

\section{Helmholtz-Manakov Solitons}

We introduce the Helmholtz-Manakov (H-M) equation as a vector generalization of the scalar Non-Linear Helmholtz (NLH) equation [2], whereby the guided electric field has two transverse orthogonal components. Exact analytical soliton solutions of the H-M equation will be derived for both focusing and defocusing media; the classic Manakov solitons are a subset of these new results. H-M solitons are found to exhibit non-trivial features that are absent from the paraxial-based descriptions (these new features will be shown to influence propagation characteristics).

\section{Stability as Robust Attractors}

Well-tested numerical perturbative techniques will be employed to demonstrate the role of H-M solitons as robust attractors (in a non-linear dynamical sense). Rich dynamical behaviour will be summarised, including evolution characteristics associated with both fixed-point (see Fig. 1) and limit-cycle attractors.
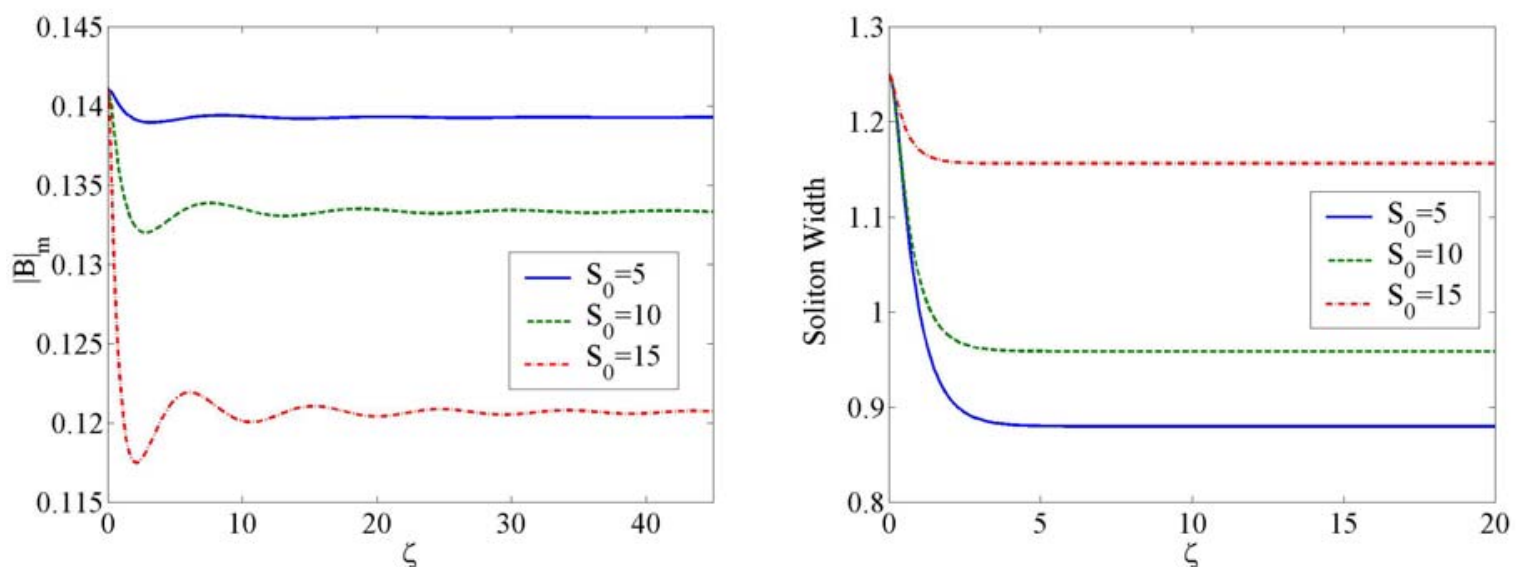

Fig. 1. Reshaping simulations using exact Manakov (paraxial) solitons as initial conditions for the H-M equation. Simulations correspond to the reshaping of (left) dark-bright, and (right) dark-dark solitons in a defocusing medium both are classed as stable fixed points.

\section{References}

[1] S.V. Manakov, "On the theory of two-dimensional stationary self-focusing of electromagnetic waves," Sov. Phys. JETP 38, 248 (1974).

[2] P. Chamorro-Posada, G.S. McDonald and G.H.C. New, "Exact soliton solutions of the nonlinear Helmholtz equation: communication," J. Opt. Soc. Am. B 19, 1216 (2002). 